\title{
Automatic Identification and Importance Evaluation Technology of Power Transmission Section
}

\author{
Weng Yixuan ${ }^{1}$, Ma Weizhe ${ }^{1}$, XieEnYan ${ }^{2}$, Qi Hui ${ }^{1}$, Liu Zhenxing ${ }^{1}$, Huang Shuang ${ }^{1}$ \\ ( ${ }^{1}$ Shenzhen Power Supply Corporation,Shenzhen 518048, Guangdong Province, China) \\ (2. Nari Technology Co., Ltd., Jiangsu Province, Nanjing, 211106)
}

\begin{abstract}
In theory, the cross section is the minimum cut set in the network. For the actual power grid, the transmission cross section is a collection of transmission lines between different regions, which is mainly used for power transmission to achieve the purpose of generating load balance. In this paper, a power grid zoning algorithm is proposed based on the topological structure characteristics and current operation state of the power grid, and the key transmission sections can be directly obtained without depending on the calculation of safety and stability margin. A practical system state partition model and key section sorting method are introduced, and a set of automatic identification method flow of key transmission sections of the system is established by searching for electrical betweenness. The importance of the transmission section is quantitatively evaluated from three angles: the margin from the stability limit, the hub position in the current power flow state, and the impact on the power grid after the power cut-off. The evaluation results can be used as a reference for decision-making in the planning and reconstruction of the transmission section, safety monitoring, protection configuration, etc.
\end{abstract}

\section{Introduction}

In recent years, several large-scale power outages occurred at home and abroad, which were caused by the failure of a weak link in the interconnected power grid and then evolved into a chain failure. Existing researches are based on the preset operation mode of power grid and its corresponding analytical laws, and their research results can not meet the requirements of online operation in scale, speed and decision-making. Interconnected tie lines between different load centers also constitute transmission channels [1]. These transmission channels have a decisive influence on the security and stability of the whole power grid, and are the key to keep the whole system running safely and stably. Therefore, some power system operation/dispatching institutions at home and abroad take the transmission section as the actual system analysis and monitoring object in order to realize dimension reduction control of large-scale power system.

Traditional transmission section selection is determined by operation mode experts based on long-term work experience and off-line analysis on the basis of geographical division [2]. As a typical complex system, some researches put forward that the complex network theory should be used to analyze the characteristics and key links of the power grid as a whole from the perspective of topological structure. If we can find out the transmission section related to overload branch, only analyze the influence degree of tripping of this branch on different branches in the section, and judge the possibility of chain overload tripping of transmission section, we can shorten the analysis time, take emergency control measures as soon as possible, and effectively avoid the chain fault caused by mechanical cutting of overload line by overload protection.

In this paper, it is proposed to construct a grid partition algorithm based on the topological structure characteristics and the current operation state of the power grid, and directly obtain the key transmission sections without depending on the calculation of safety and stability margin. In addition, combined with the relevant principles and requirements of section safety in actual power grid operation, the importance of important transmission sections of UHV AC/DC power grid in the future planning year in China is emphatically studied.

\section{Basic Concept of Transmission Section}

Usually, the transmission section in the actual system is formed by the system dispatcher selecting a group of tie lines connected to the same power supply area and load area according to the geographical location and operation experience. The power flow transfer factor is only related to the structure and parameters of the power network, and can measure the influence of the disconnected branch on the power flow of the surrounding branches. However, for the transmission section composed of electromagnetic ring network, there may be some deviation between the monitoring objects and targets determined by this analysis method and the actual monitoring requirements of power 
system [3]. Transmission sections can be classified in different ways. A common classification method is to classify according to whether the voltage levels in the same transmission channel are the same, including multiple sections with different voltage levels which are electromagnetic ring network sections, and sections with only one voltage level which are non-electromagnetic ring network sections.

Transmission section embodies the fragile link of the power grid, which will have an important impact on the power transmission at both ends and directly threaten the safe and stable operation of the power grid. Under a certain operation mode, a set of transmission lines with the same active power flow direction and close electrical distance in the system. It should be pointed out here that the same direction of active power flow is not a strict constraint. Transmission cross-section identification is to find out the branch set with large power flow transfer factor after a branch is cut off, that is, the branch set with large transfer current in the equivalent network. In this case, the transmission capacity of the whole section is mainly restricted by the branches with low voltage level. These branches which restrict the transmission capacity of the whole section are defined as the "key branches" of the section in this paper. Most of them are not needed in practice, so it is necessary to make full use of the above characteristics of the section to simplify it, and then search the section.

\section{Fast Identification Method of Transmission Section based on Cutting Point and Path Search}

\subsection{Transmission section search based on partition section.}

After the power grid is partitioned, a tree diagram of community structure decomposition can be obtained, that is, hierarchical clustering tree. Each station includes multiple voltage classes and transformers, and there are no lines in the station, so the transmission section in the actual power grid cannot appear in the station, so all nodes in a station can be merged into one point. Firstly, increase the load power in the receiving area of the cross section, and correspondingly increase the unit output in the power transmission area of the cross section according to a certain dispatching strategy, and repeat this process until a certain constraint is broken.

Usually, after the transmission section of the system is determined, it will be analyzed by static $\mathrm{N}-1$, so for each line in the section, it must be the most closely connected group of lines under the static N-1 security constraint [4]. When a branch in the section is cut off, the branch with the largest cut-off distribution coefficient in the whole power grid must also exist in the section; Continue to break the second branch in the section, and the line corresponding to the calculated maximum branch break distribution coefficient is still contained in the section, and so on, until the whole network is divided into two independent parts, thus a transmission section can be obtained (Figure 1).

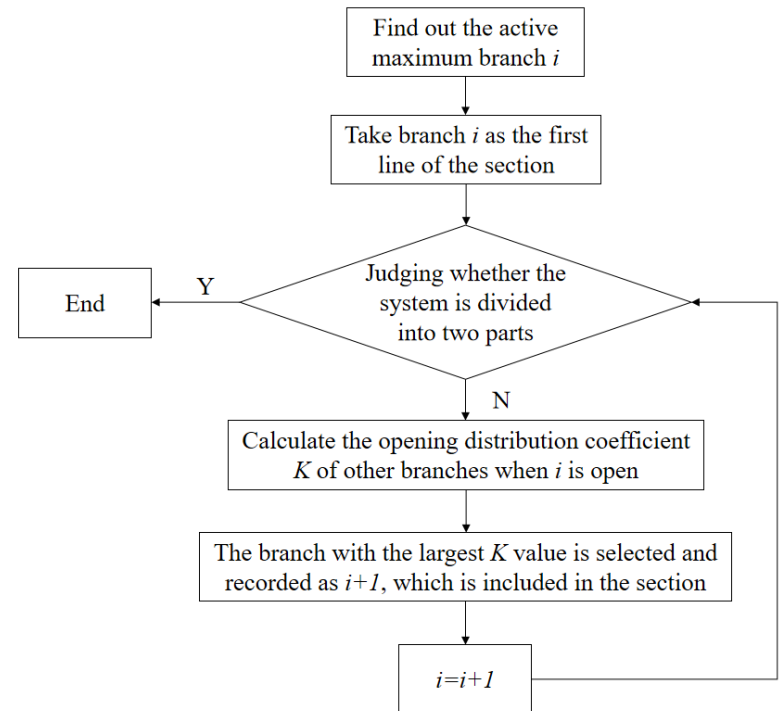

Figure 1 Flow chart of branch break distribution coefficient method

Transmission cross-section identification is to find out the branch set with large power flow transfer factor after a branch is cut off, that is, the branch set with large transfer current in the equivalent network. The transmission lines and transformer branches are abstracted as edges, and then the key nodes and fragile lines in the system are identified based on a series of indexes such as average path length, node degree, clustering coefficient and intermediate number. And is connected with one or more high-voltage buses through a substation. For high-voltage section, the line cannot appear in the power supply area, so one power supply area can be combined into one point.

\subsection{Searching and sequencing of transmission sections by using electrical betweenness.}

Transmission section embodies the weak links in the system, and is usually composed of weak lines in the system, which is of great significance to the safe and stable operation of the system. A group of branches represented by tie lines between regions constitute transmission channels, which generally have the characteristics of large transmission capacity, high voltage level and consistent power flow direction. For any contracted power supply area, there are usually several power transmission lines and several power transmission lines, all of which may be part of the transmission section. High transmission dielectric lines play an important role in power transmission and maintaining the security and stability of the power grid, thus ensuring that the sectional area meets the characteristics. One is to judge whether there is a close electrical relationship between a branch and the leading branch, and the qualified branch is regarded as the cross-section alternative branch; the other is to judge whether the leading branch and the cross-section alternative branch meet the constraint of mutual parallelism, and eliminate the alternative branches that do not meet the parallel conditions.

The line in the transmission section is responsible for transmitting power from the power supply area to the load area. When a power transmission path between the power supply area and the load area is disconnected, the original 
transmission power will be transferred to other power transmission paths for transmission; These branches constitute the transmission section of overload branch, that is, the power flow transfer transmission section. When the two branches only have the same power supply area or load area, the occurrence of power flow transfer is often accompanied by the system operation state changes such as the output regulation of generator sets and the reverse of power flow. After a branch is cut off, the transmission medium of a line with large power flow transfer distribution factor also increases greatly, which will easily become a cut-off line in further cut-off.

As shown in fig. 2, the system is regarded as a linear network. according to the superposition principle, switching off the branch $L_{m n}$ is equivalent to superimposing an equivalent network as shown in fig. 2(b) on the basis of the original network, that is, both ends of $L_{m n}$ are connected with a reverse current source with current $I_{m n[5] \text {. }}$



(a) (b)

Figure 2 Schematic diagram of equivalent superposition network after branch disconnection

According to the equivalent superposition network shown in fig. 1(b), the current increment generated by opening branch $L_{m n}$ on branch $L_{i j}$ is

$$
\Delta I_{i j}=\frac{X_{i j-m n} / x_{i j}}{1-X_{m n-m n} / x_{i j}} I_{m n}=\frac{X_{i m}+X_{j n}-X_{i n}-X_{j m}}{X_{i j}-\left(X_{m m}+X_{n n}-2 X_{m n}\right)} I_{m n}
$$

$x_{i j}$ is the impedance value of branch $L_{i j}, X_{i j-m n}$ is the mutual impedance between node pairs $i$ and $j$ and node pairs $m$ and $n, X_{m n-m n}$ is the self impedance of node pairs $m$ and $n, X_{i j}$ is the element of system node impedance matrix $[X]$.

Let $\lambda_{e}$ be the opening and closing distribution coefficient of branch ${ }^{m n}$ to branch ${ }^{i j}$, which is defined as

$$
\lambda_{e}=\frac{\Delta I_{i j}}{I_{m n}}=\frac{X_{i m}+X_{j n}-X_{i n}-X_{j m}}{x_{i j}-\left(X_{m m}+X_{n m}-2 X_{m n}\right)}
$$

It can be seen from formula (2) that the system is regarded as a linear network in the calculation process, and the equivalent DC power flow model is adopted. Therefore, the calculated branch break distribution coefficient is actually the current transfer coefficient, which is only related to the structure and parameters of the power grid, and the maximum error between the calculated result and the actual power flow transfer coefficient can reach $10 \%$ [6].

\subsection{Search for closed loop of power transfer.}

The main branch is added as the first branch in the cross-section alternative branch set, and all the cross-section alternative branches are sorted according to the absolute value of the opening and closing distribution coefficient of the main branch, and the higher the coefficient, the higher the sorting order is; If the new transmission link is radial, it will be directly used as the key transmission section, and the line with the highest importance in the transmission link will be used as the leading line of the section; For several adjacent power supply areas with connections, ignoring the connection lines between them and merging them into a larger power supply area, all the power transmission lines or transmission lines may form a complete section. Because the regional tie line is a branch with weak connection between nodes in the system, the regional tie line of power grid can be determined according to the electrical distance between adjacent node pairs. The setting of the preset threshold value follows the following principles: the proportion of the selected key branches representing the line vulnerability to the total number of branches in the system does not exceed $30 \%[7]$.

As shown in Figure 3, when the curve reaches the critical point, the whole task of state deduction and power flow calculation is completed. By analyzing the system state (node voltage and power angle) at this time, the limit transmission capacity of the system can be obtained.

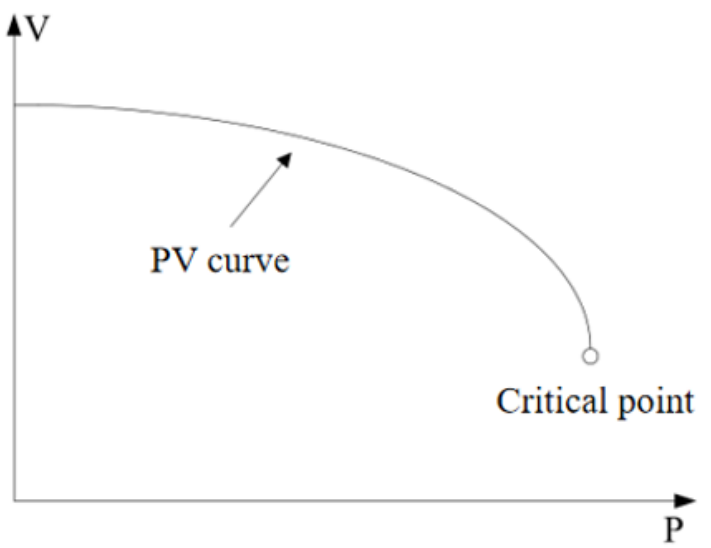

Figure 3 Schematic diagram of PV curve

When the PV curve approaches the critical point, the voltage collapse index $I C$ used to evaluate the main eigenvalue of Jacobian matrix will approach 0 [8]. The voltage collapse index $I C$ is defined as follows:

$$
I C=T V^{t} * J * T V
$$

In the formula, $T V$ represents the tangent phasor of state deduction, superscript $t$ represents the transposition of phasor, and $J$ is Jacobian matrix. Define the end criterion of state deduction as follows:

$$
I C \leq I C
$$


In the formula, $I C_{\text {set }}$ is the threshold value of voltage collapse index. When the voltage collapse index IC drops continuously and is smaller than the threshold value, it indicates that the current system state can be approximated as a critical point state, and the whole state deduction process can be finished.

Carry out network topology analysis to judge whether the system is divided into two independent networks; If yes, confirm that it is a transmission section, and go to the next search; By calculating the safety and stability margin to analyze the importance of the section, the result is more accurate, but the amount of calculation is greatly increased. The composition of cross-section only depends on the close degree of electrical relationship between branches, and is not limited by the geographical and regional restrictions of general electrical partitions: when two power transmission paths intersect to form a closed loop, other nodes in the two paths should be the same except the nodes forming the closed loop.

\section{Importance Evaluation of Transmission Section Based on Power Grid Performance Change}

In order to quantitatively evaluate the dependence of the power grid on different transmission sections, the most direct way is to investigate the changes of the basic performance of the power grid before and after all branches of the transmission section are cut off. Because power flow tracking needs to update units and load sets according to power flow changes, the calculation speed is slow. However, the calculation speed of sensitivity factor method based on DC power flow model is fast. Considering the safe and stable operation of incomplete grid in the transition stage of power grid construction, a single-channel transmission scenario is first drawn up based on the initial grid in the planning period to analyze the safety and stability of the transmission section. The line part included in the section may also belong to the first type of key section, which is not quite consistent with the key section that normal people care about. This section can be considered as an extension of the key section, and it is also worthy of attention.

On the premise of ignoring the network loss, the total amount of power transmission in the power grid can be characterized as the total amount of active load in the power grid The diversity of power transmission modes can be characterized by the number of power transmission paths in the power grid, and the calculation formula of power transmission efficiency $E_{\text {grid }}$ is defined as formula (5):

$$
E_{\text {grid }}=\frac{\sum_{i \in G, j \in L ; i \neq j} M(i j) \cdot \sum_{j \in L} P_{j}}{\sum_{i \in G, j \in L ; i \neq j} \sum_{m=1}^{M(i j)} w_{i j}(m) d_{i j}(m)}
$$

In the formula, $i$ and $j$ are node numbers; $G$ is the generation node set in the power grid, and $L$ is the load node set in the power grid; $M(i j)$ is the number of power transmission paths between the power generation node $i$ and the load node $j ; P_{j}$ is the active load of load node $j$; $w_{i j}(m)$ is the weight coefficient of the mth electric energy transmission path between generating node $i$ and load node $j$, that is, the active power transmitted on this path; And $d_{i j}(m)$ is the path transmission distance of the $m$ th electric energy transmission path between the generating node $i$ and the load node $j$.

Setting the partition end condition as that there are no more disconnectable lines in the system, so as to obtain the key transmission sections corresponding to all transmission links of the system. When selecting control nodes, the output nodes are determined according to the sensitivity coefficient of nodes to overload lines, but the sensitivity value is related to the impedance and the selection of reference nodes. However, in reality, the attenuated DC component is only a physical DC component. After Fourier transform, it can be extended to mathematical components with different frequencies, including fundamental frequency components. The transmission lines and transformer branches are abstracted as edges, and then the key nodes and fragile lines in the system are identified based on a series of indexes such as average path length, node degree, clustering coefficient and intermediate number. Specifically, the power transmission range, total power transmission amount, power transmission mode and power transmission efficiency of the power grid should be comprehensively considered in the change of power grid performance.

In order to illustrate the advantages of the proposed method, the method in this paper (hereinafter referred to as "Method 1") and the method of calculating the limit power of the section by directly taking the searched section branch as the control object (hereinafter referred to as "Method 2") are used to analyze the control object and limit value in the section direction respectively. The power flow of some related branches obtained by these two methods is shown in Table 1.

Table 1 The power flow of some branches related to critical points is calculated by two methods

\begin{tabular}{ccccccc}
\hline \multirow{3}{*}{ Branch } & \multicolumn{3}{c}{ Method 1 } & \multicolumn{3}{c}{ Method 2 } \\
\cline { 2 - 7 } & $\begin{array}{c}\text { Ground } \\
\text { state }\end{array}$ & $\mathrm{N}-1$ & Safety & Ground & $\mathrm{N}-1$ & Safety \\
& tidal & trend / & margin & state & trend / & margin \\
& current & M W & $/ \%$ & current & M W & $/ \%$ \\
\hline
\end{tabular}




\begin{tabular}{ccccccc}
\hline \multicolumn{7}{c}{$/ \mathrm{M} \mathrm{W}$} \\
\hline$l_{D_{2}-D_{1}}$ & 1753.01 & 2401.37 & 30.14 & 2796.55 & 2832.18 & 23.41 \\
\hline$l_{D_{3}-D_{1}}$ & 1583.72 & 2236.08 & 24.75 & 268413 & 2719.65 & 10.17 \\
\hline$l_{A-H}$ & 451.24 & 674.18 & 0.96 & 501.49 & 667.82 & 12.88 \\
\hline$l_{D-H}$ & 289.33 & 554.22 & 17.44 & 488.72 & 510.37 & 0.73 \\
\hline$l_{E-H}$ & 241.70 & 417.85 & 28.91 & 358.65 & 418.52 & 19.47 \\
\hline
\end{tabular}

It can be seen from Table 1 that in the identification of transmission section, if the critical branch is not taken into account and the branch set $\left\{l_{D_{2}-D_{1}}, l_{D_{3}-D_{1}}, l_{D-H}, l_{E-H}\right\}$ is directly used as the analysis and monitoring section, the safety level of the system will not be accurately measured, which may lead to the deviation of the control decision of the dispatcher, and then lead to the system safety problem.

Transmission section embodies the weak links in the system, and is usually composed of weak lines in the system, which is of great significance to the safe and stable operation of the system. The control nodes used to eliminate unbalanced power can be sensitive to overload lines (existing sensitivity methods) or very sensitive to overload lines (adjusting the output of remote control nodes). Therefore, the fragile lines in the system can be identified, and the key lines meeting certain requirements can be screened out. Generally speaking, the higher the line electrical dielectric value, the more important the line plays in the transmission of active power flow. There is no change in the ranking results of key transmission sections. The following conclusions can be drawn: the comparison algorithm pays more attention to the structural vulnerability of power grid, but it cannot accurately reflect the change of power flow distribution caused by the change of operation mode, while the algorithm in this paper can comprehensively reflect the power grid vulnerability determined by both.

\section{Summary}

It is the future development trend of power grids in various countries to realize the optimal allocation of resources through the interconnection of regional power grids. In this paper, it is considered that the key to effectively deal with or even eliminate this hidden danger lies in accurately identifying the weak key components in the system power grid, searching and identifying the component sets closely related to these key components, identifying the key links and key transmission sections in the grid and reasonably evaluating their importance. Introducing critical branch identification into automatic cross-section discovery is helpful to provide dispatchers with monitoring objects and targets that can accurately reflect the safety characteristics of the system. According to the connection cut set between regions and the consistency characteristics of power flow, the transmission section identification method based on the consistency characteristics of power flow in transmission channels is summarized Based on the research of these two transmission section identification methods, a multi-angle analysis system of transmission section is established.

\section{References}

1. Xu Yan, Zhi Jing, Wang Xianghui. Evaluation of thermal stability power limit of transmission section based on N-1 principle[J]. Journal of Electric Power System and Automation, 2017(29):17.

2. He Peiying, Fang Xinyan. Fast search of key transmission sections based on clustering algorithm[J]. Power System Protection and Control, 2017, 045(007):97-101.

3. Wang Ziqi, He Jinghan, Zhang Dahai, et al. Complex network transmission section identification method based on current transfer proportional coefficient[J]. Power System Technology, 2017(03):245-252.

4. Zhu Bin, Qiu Lijun, Wang Yong, et al. On-line calculation method of dynamic thermal stability limit of power grid transmission section[J]. Power System Protection and Control, 2017, 45(003): 94-99.

5. Gan Deshu, Lin Guihui, Wu Haixiong, et al. Power grid dispatching operation control system and system development centered on section control[J]. Power System Protection and Control, 2017(22):117-124.

6. He Jinghan, Wang Ziqi, Zhang Dahai. Transmission section identification method based on network segmentation and side path search [J]. Electric Power Automation Equipment, 2017, 37(007):1-7.

7. Lu Junyu, Lin Shenghong, Mao Chizu, et al. Identification of key transmission sections based on community discovery theory [J]. Shaanxi Electric Power, 2017, 045(008): 51-57.

8. Wang Wenyuan, Li Weiguo, Cheng Yudian, et al. Method for determining key cross-sections based on key branches[J]. Journal of North China Electric Power University (Natural Science Edition), 2018, 45(002): 54-63. 